\title{
LIQUID ARGON CALORIMETER PERFORMANCE AT HIGH RATES
}

\author{
VICTOR KUKHTIN \\ Joint Institute for Nuclear Research \\ Joliot-Curie 6, 141980 Dubna Russian Federation \\ E-mail:kukhtin@sunse.jinr.ru
}

on behalf of the HiLum ATLAS Liquid Argon Endcap collaboration

\begin{abstract}
The performance of the ATLAS liquid argon endcap and forward calorimeters has been projected at the planned high luminosity LHC option HL-LHC by exposing small calorimeter modules of the electromagnetic, hadronic, and forward calorimeters to high intensity proton beams at IHEP/Protvino accelerator. The results of HV current and of pulse shape analysis, and also the dependence of signal amplitude on beam intensity are presented.
\end{abstract}

Keywords: LHC, LH-LHC, LAr calorimetry, space charge effect

\section{Introduction}

The increase of instantaneous and integral luminosity by a factor of up to 5 with respect to the nominal LHC value has serious consequences for the signal reconstruction, operation and radiation hardness requirements of the electromagnetic endcap (EMEC) [1], hadronic endcap (HEC) [2], and forward (FCal) [3] calorimeters. Small modules of each calorimeter type have been build , placed in separate cryostats, and exposed to high intensity proton beams at IHEP. By placing absorber elements in-between the cryostats the ratios of particle and energy flaxes have been adapted to the ATLAS situation. In liquid argon calorimeters at high enough ionization rates space-charge effects, instigated by the build-up of the slowly drifting positive argon ions, can degrade the signal. The impact of the space-charge effects on the calorimeter signal e and on the high voltage (HV) current are discussed. 


\section{Set-up, beam and be am instrumentation}

The general set-up of the three calorimeter modules in the beam ( see Fig.1) has been optimized based on the results of the detailed Monte Carlo studies.

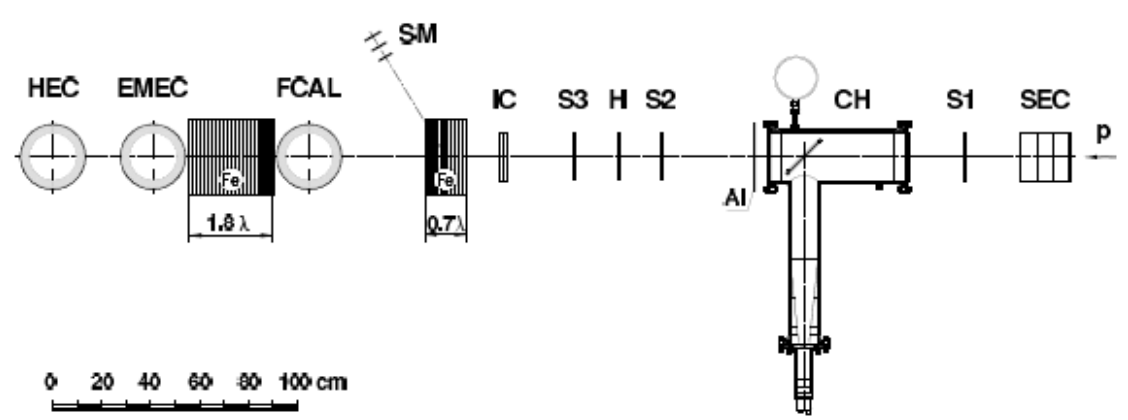

Figure 1. Schematic top view of the overall test beam set-up. Shown are the three cryostats with the FCal, EMEC and HEC module and the related Fe absorbers as well as the beam instrumentat ion: the scintillation counters $\mathrm{S} 1, \mathrm{~S} 2, \mathrm{~S} 3$ and the scintillation counter hodoscope $\mathrm{H}$. The beam intensity is monitored with the secondary emission chamber SEC, The Cerenkov counter $\mathrm{CH}$, the ionization chamber IC, the scintillation count er monitor SM and the aluminium foil Al

The proton beam of $50 \mathrm{GeV}$ is extracted from $\mathrm{U}-70$ synchrotron via channeling with bent crystal technique [4]. The accelerator has been operated with only 5 out of 30 bunches filled with the spacing between filled bunches almost $1 \mu \mathrm{s}$. It allows to study the high flux response of the calorimeters not affected by pile-up from $\mathrm{m}$ revious bunches. Using the maximum deposited energy density fro $\mathrm{m} \mathrm{MC}$ minimum bias events in ATLAS for endcap calorimeters and the corresponding numbers for IHEP beam setup for one incident proton the correspondence between LHC luminosity and beam intensity was found for each modules. It is estimated that the uncertainty in the numbers is at least at the level of a factor two.

\section{Cal orimeter modules}

A replica of a small section of electromagnetic module of the ATLAS FCal (see Fig.2) is $50 \mathrm{~mm}$ deep with two groups of 16 electrodes each. One group has $119 \mu \mathrm{m}$ liquid argon gap width with HV potential $100 \mathrm{~V}$ applied, just prepared to test proposed for the upgraded ATLAS FCal at the HL-LHC, 

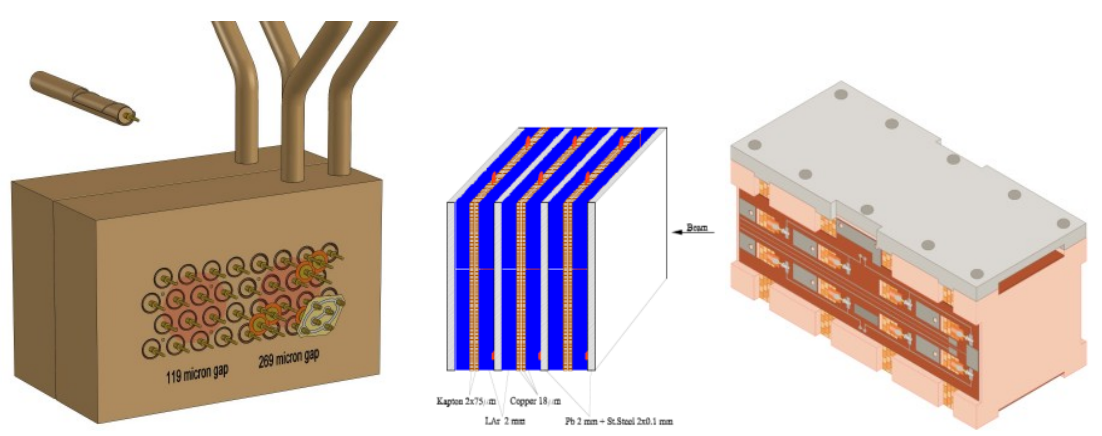

Figure 2.Schematic view of calorimeter modules installed each in separate cryostat (from the left to the right) - FCal with two different liquid argon gap sizes, EMEC and HEC.

Either half of the FCal module can be centered on the beam to study each option individually.

The EMEC and HEC modules are both of planar structure(rather than the accordion geometry for the EMEC) with the argon gap width $2 \mathrm{~mm}$. The positive $\mathrm{HV}$ up to $2 \mathrm{kV}$ was applied to both modules.

The read-out electronics is rather close to that used in the early ATLAS HEC test beam tests at CERN. But instead of operating preamplifiers in cold as for HEC in ATLAS, warm preamplifiers were mounted on the front-end board located outside the cryostat. The total number of read-out channel for all three modules was equal to 16 .

\section{Results of $\mathbf{H V}$ current analysis}

Charged particles, passing through the LAr gaps of the calorimeter and producing drifting ions and electrons, induce also a DC current in the $\mathrm{HV}$ system. This current is correlated with the average beam intensity, resp. the luminosity at LHC or HL-LHC. An additional to the standard HV supply system external measurement device [5] has been constructed. The logging rate of this device is at $10 \mathrm{~Hz}$ per channel and the precision of the time stamp is $10 \mathrm{~ms}$. It is connected either to the FCal or EMEC HV supply system. Electronic noise limits the effective resolution to about $25 \mathrm{nA}$. Shown at Fig. 3 FCal HV current ) behaviour as function of proton beam intensity was fitted by the straight line. The non-linear contribution was estimated to be less that $0.36 \%$ at a beam intensity corresponding to the nominal LHC lu minosity. 

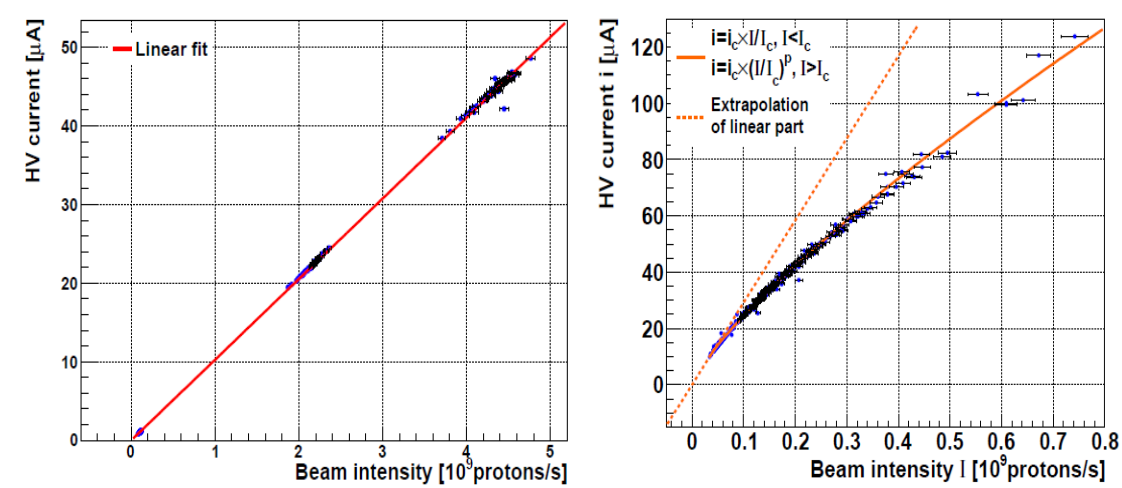

Figure 3. Total FCal ( on the left ) and EMEC ( on the right) HV current as function of the beam intensity. The beam measurements were dine by ionization chamber for $\mathrm{FCal}$ and Cherenkov counter for EMEC.

With the larger liquid argon gap it is expected that the linear relation between the beam intensity and $\mathrm{HV}$ current to change to a power dependence as it is seen for the EMEC on Fig.3 .

\section{Dependence of the pulse shape and the signal amplitude on beam intensity}

To compare the signals, data were taken at each beam intensity varying the HV setting for calorimeter modules. For modules with larger LAr gaps at low intensity the difference in signal length due to the variation of the electron drift velocity with the electric field is found. The further reduction of length for intensities above the critical value follows closely the expectation from the space-charge simulation.

The dependence of the signal amplitude on the beam intensity has been studied for all modules and for EMEC and FCal-100 modules shown at Fig. 4. 

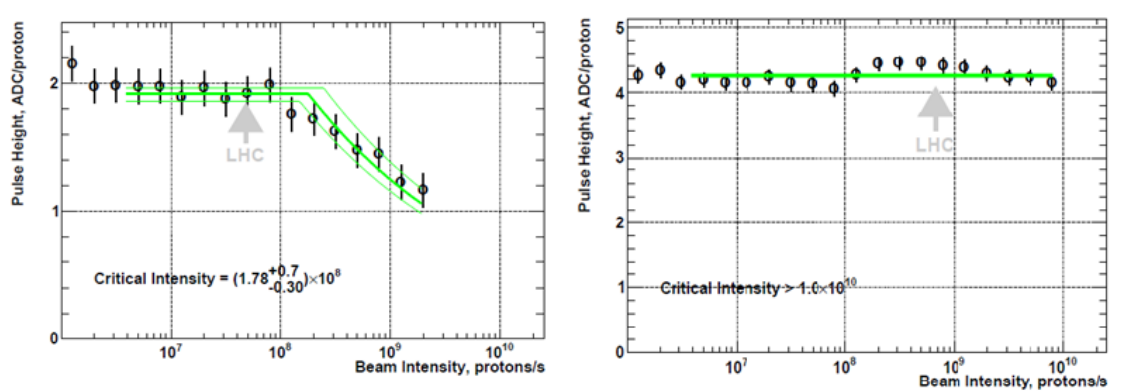

Figure 4 Dependence of pulse height of HEC (on the left) and FCal (119) (on the right) modules on beam intensity

A model for small bulk recombination rate constant assumes constant response below the critical intensity and a falling power dependence above. The data for the $119 \mu \mathrm{m}$ LAr gap FCal module do not show any dependence on beam intensity

\section{Conclusions}

The observed pulse shapes follow closely the prediction of simulation. The space-charge effects are visible in the signal amplitude for the calorimeter modules with larger LAr gap size but they are absent for $119 \mu \mathrm{m}$ gap width. The dependence of the HV current on the beam intensity is in agreement with the expectations.

\section{References}

1. M. Aleksa et al., Construction, assembly and tests of the ATLAS electro magnetic end-cap calorimeters, JINST 3 (2008) P06002..

2. D.M. Gingrich et al., Construction, Assembly and Testing of the ATLAS Hadronic End-Cap Calorimeter, JINST 2 (2007) P05005.

3. A.Artamonov et al., The ATLAS Forward Calorimeter, JINST 3 (2008) P02010.

4. V.I.Kotov et al., Application of bent crystals at IHEP $70 \mathrm{GeV}$ accelerator to enhance the efficiency of its usage, Proc. of EPAC 2000, Vienna, Austria, pp.364-366.

5. A.Afonin et al., Relative luminosity measurement of the LHC with the ATLAS forward calorimeter, JINST 5 (2010) P05005. 